\title{
Delayed mutual information infers patterns of synaptic connectivity in a proprioceptive neural network
}

\author{
Wagner Endo • Fernando P. Santos • David Simpson • \\ Carlos D. Maciel • Philip L. Newland
}

Received: 29 September 2014 / Revised: 12 January 2015 / Accepted: 21 January 2015 / Published online: 3 February 2015

(C) Springer Science+Business Media New York 2015

\begin{abstract}
Understanding the patterns of interconnections between neurons in complex networks is an enormous challenge using traditional physiological approaches. Here we combine the use of an information theoretic approach with intracellular recording to establish patterns of connections between layers of interneurons in a neural network responsible for mediating reflex movements of the hind limb of an insect. By analysing delayed mutual information of the synaptic and spiking responses of sensory neurons, spiking and nonspiking interneurons in response to movement of a joint receptor that monitors the position of the tibia relative to the femur, we are able to predict the patterns of interconnections between the layers of sensory neurons and interneurons in the network, with results matching closely those known from the literature. In addition, we use cross-correlation methods to establish the sign of those interconnections and show that they also show a high degree of similarity with those established for these networks over the last 30 years. The method proposed in this paper has great
\end{abstract}

Action Editor: Catherine E Carr

\section{W. Endo}

Department of Electrical Engineering, Federal Technological University of Paraná, Av. Alberto Carazzai, 1640 Cornélio

Procópio, Paraná, Brazil

W. Endo $(\varangle) \cdot$ F. P. Santos $\cdot$ C. D. Maciel

Signal Processing Laboratory, Department of Electrical Engineering, University of São Paulo, Av. Trabalhador São Carlense, 400 São

Carlos, São Paulo, Brazil

e-mail:wendo@utfpr.edu.br

D. Simpson

Institute of Sound and Vibration Research, University of

Southampton, University Road, Highfield, S017

1BJ Southampton, UK

P. L. Newland

Centre for Biological Science, University of Southampton, Highfield

Campus, S017 1BJ Southampton, UK potential to elucidate functional connectivity at the neuronal level in many different neuronal networks.

Keywords Delayed mutual information · Information flow · Interneuron $\cdot$ Locust limb control $\cdot$ Reflex

\section{Introduction}

Knowing the structural connectivity of neural networks in the brain provides us with an understanding of their function and interaction, however establishing maps of connectivity using traditional physiological recording approaches is a technically demanding and time consuming process (Bressler and Seth 2011; Stetter et al. 2012). Moreover, sampling bias is inevitable as we can never fully reveal the connections of one neuron with all of its presynaptic and postsynaptic targets. While connectomics aims to understand the connectivity of neurons, synapses and pathways in the brain using anatomical techniques, relatively new statistical approaches have been proposed to model neurobiological systems. Using these methods the connectivity can be inferred from the activity of individual neurons and their directional interactions to provide a deeper insight of the function of neurons and pathways, and the underlying processes that drive the networks (Sommerlade et al. 2011). These statistical approaches, however, are often based on surrogate or simulated data with few focusing on real neural networks (Gerhard et al. 2013) or how good such models are at predicting, or inferring, their neuronal interactions.

The analysis of signals measured from such complex biological systems has many methodological challenges (Müller et al. 2003), mostly due to the non-linear relationships between input and output, and the stochastic nature of neural responses. Under these circumstance the usefulness of 
techniques based on conventional analysis (including spectral) of time series tends to limit the understanding of the biological system (Mars and van Arragon 1982) and hence different approaches have been sought. A number of studies have investigated the causal relationships, or influences, between signals in order to quantify the strength of interaction (Hlavácková-Schindler et al. 2007; Shibuya et al. 2009). Many causality measures have been proposed that make possible the explicit evaluation of a causal relationship between signals (Pearl 2009). In particular, Wiener-Granger Causality has been commonly used in neuroscience even though such causality measures only capture the linear features of time series data (Bressler and Seth 2011), and that there is a requirement for stationarity in the time series; something that seldom occurs in neural signals (Dewhirst et al. 2013).

An alternative approach to analyze non-linear and stochastic signals is based on information theory (Bialek et al. 2001; Cover and Thomas 2006; Schreiber and Schmitz 2000; Shannon 1948). In particular, mutual information has been used as a measure of the statistical dependency between two variables and may be interpreted as a generalization of the correlation of non-linear relationships between multiple processes with arbitrary probability distributions (Erdogmus and Principe 2006; Li 1990). The main limitation to mutual information is its inability to differentiate the association direction between two signals (Schreiber 2000). To overcome this limitation Schreiber (2000) introduced a quantity called information transfer that shares some of the desired properties of mutual information but takes into account the dynamics of information transfer. Such an approach is now widely used to analyse data from econometrics (Marschinski and Kantz 2002) to biomedical engineering (Jin et al. 2010; Li and Ouyang 2010; Ward and Mazaheri 2008).

In general information theoretic approaches make no assumptions about the relationship among the system variables (e.g., strict stationarity, Nichols 2006). Timedelayed mutual information quantifies the co-dependence between variables by considering shared previous information content as a function of time (Nichols et al. 2005; Palus et al. 2001; Vastano and Harry 1988). Here we analyse the delayed mutual information (DMI) estimated from a large multivariate neurobiological data set obtained by Angarita-Jaimes et al. (2012), Kondoh et al. (1995), Newland and Kondoh (1997a,b) and Vidal-Gadea et al. (2010) from a neural network that produces and controls movements of the hind leg of an insect, the desert locust. We utilize the nervous system of the locust for analysis due to its relative simplicity, accessible nervous system and extensive background of physiological and morphological studies (see Burrows 1996). We establish the time delays in mutual information and channel capacities of interneurons in different layers of a proprioceptive network and ask whether they can be used as predictors of synaptic connectivity.

\section{Material and methods}

Adult male and female desert locusts, Schistocerca gregaria (Forskål) were used for all experiments. Locusts were mounted ventral-side-uppermost in modelling clay and the apodeme of the $\mathrm{FeCO}$ exposed by opening a small window of cuticle in the distal anterior femur (Kondoh et al. 1995), grasped between the tips of fine forceps attached to a shaker (Ling Altec 101) and cut distal to the forceps. The metathoracic ganglion was exposed by making a small window in the ventral thorax, supported on a wax covered silver platform, and the sheath treated with protease (Sigma type XIV) for $1 \mathrm{~min}$ before recording. Microelectrodes filled with potassium acetate and with DC resistances of $50-80 \mathrm{M} \Omega$ were driven through the sheath and into the neuropilar processes of the nonspiking local interneurons, the axons of sensory neurons, or the somata of spiking local interneurons and intracellular recordings (Fig. 1) made using an Axoclamp 2A amplifier (Axon Instruments, USA).

The forceps holding the chordotonal organ apodeme were moved with a GWN (Gaussian White Noise) signal produced by filtering a pseudorandom binary sequence (CG-742, NF Circuit Design Block) band-limited to $27 \mathrm{~Hz}$ with low-pass filters (SR-4BL, NF Circuit Design Block) with a decay of $24 \mathrm{~dB} /$ octave (Fig. 1c,d). This generated a GWN signal that had a Gaussian probability density function in the bandwidth of interest. Stimulus and evoked responses of the interneurons were stored on magnetic tape using a PCM-DAT data recorder (RD-101 T, TEAC, Japan). Subsequently, all signals were sampled at a rate of $10 \mathrm{kHz}$ offline to a personal computer for later analysis.

\subsection{Analysis}

The algorithms were developed in Python 2.7 running on a Linux (Ubuntu 13.10) i7 computer with the code utilising the resources of parallel programming in the IPython environment (Pérez and Granger 2007).

The data set consisted of recordings from 36 different nonspiking, and 25 spiking, local interneurons and 12 sensory neurons. The responses of all neurons were evoked by stimulation of the $\mathrm{FeCO}$ with a GWN band limited to $27 \mathrm{~Hz}$ for at least 40s applied to the $\mathrm{FeCO}$ apodeme. Only the stationary segment following an initial transient at the onset of stimulation was analyzed (Dewhirst et al. 2013).

The estimation of probability density used a histogram approach, while for joint probability distributions bidimensional histograms were used. In both cases the number of bins was fixed at 32 and their length depended on the range of variables under analysis. The DMI and mutual information were calculated for a range of lag values. 
Fig. 1 Neural network underlying reflex movements of the tibia of a hind leg. a. The $\mathrm{FeCO}$ in the distal femur is displaced when its apodeme is stretched and relaxed by tibial movements. b. Sensory neurons in the $\mathrm{FeCO}$ send axons to neural networks in the metathoracic ganglion where signals are processed (modified from Burrows 1996). c. A spiking local interneuron receives excitatory inputs and spikes during displacement of the $\mathrm{FeCO}$ apodeme with GWN. d. A nonspiking local interneuron receives a barrage of large depolarising excitatory potentials in parallel with depolarisation of the slow extensor motor neuron (SETi) and the posterior slow flexor tibiae motor neuron during displacement of the apodeme with Gaussian White Noise. $\mathbf{c}$ and d are from different animals
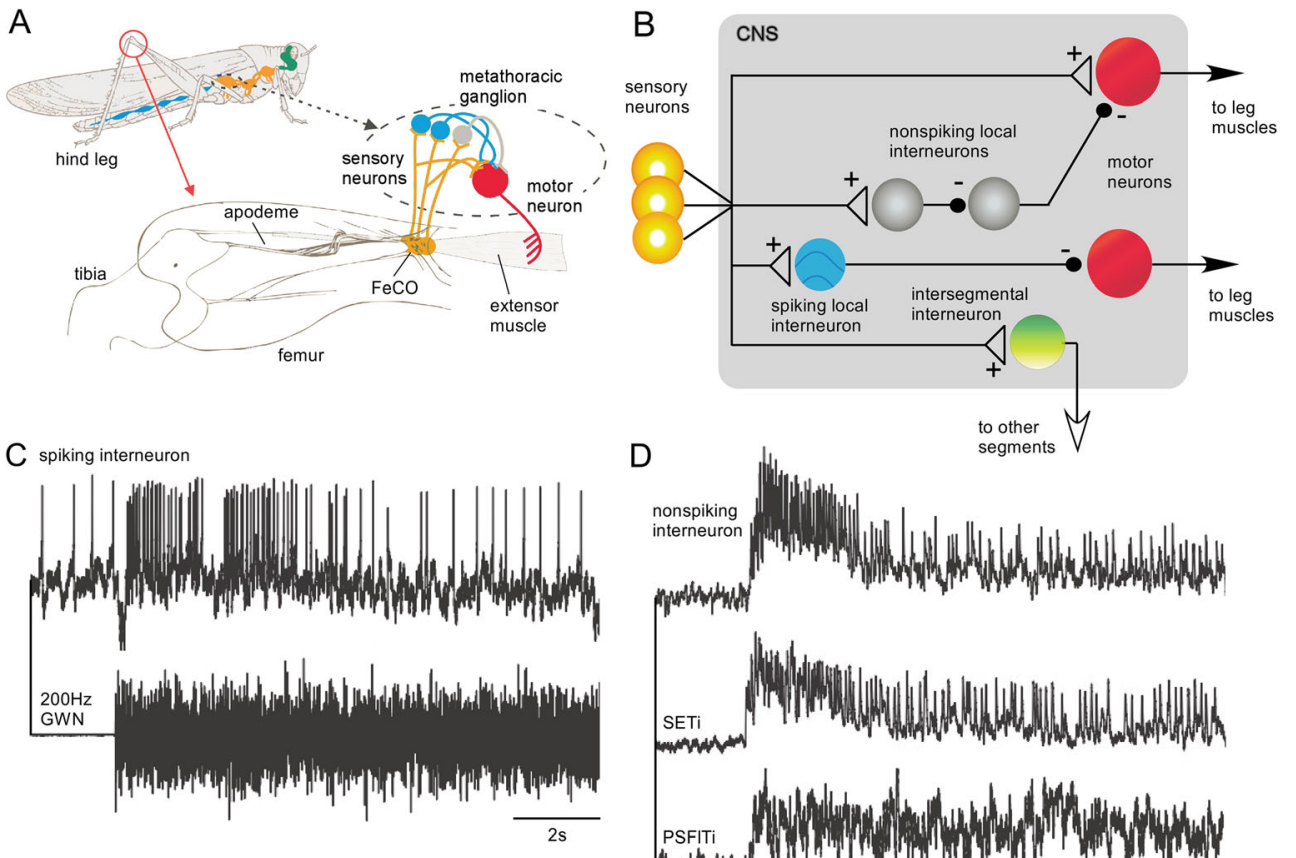

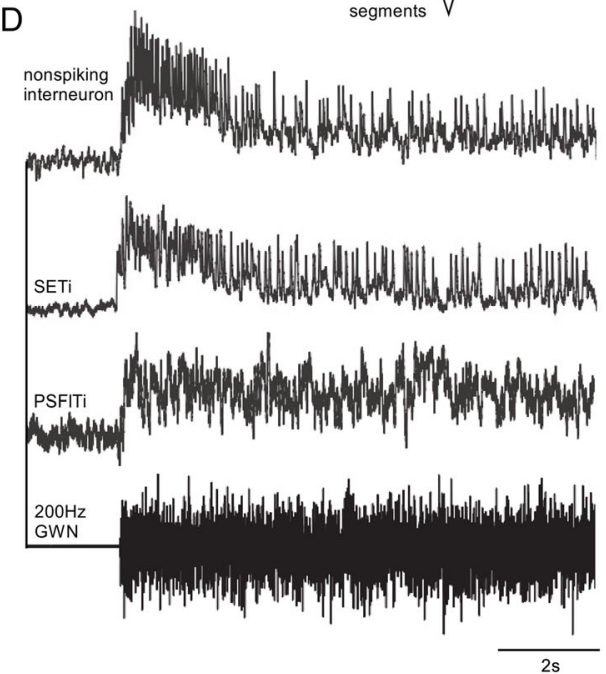

\subsection{Association measurements}

Mutual information (MI) is a measure of the association between two random variables (Cover and Thomas 2006; Dionisio et al. 2004) and quantifies the shared information based on measures of uncertainty given the conditional entropy of the two variables. The equation for MI is described as follows (Shannon 1948):

$\begin{aligned} I(X ; Y) & =H(Y)-H(Y \mid X) \\ & =H(X)+H(Y)-H(X ; Y)\end{aligned}$

where $H(Y \mid X)$ is the conditional entropy of $\mathrm{Y}$ given $\mathrm{X}$.

To evaluate MI we used an estimate of the probability density function of the signals described by the following Eq. 2 (Cover and Thomas 2006):

$I(X ; Y)=\sum_{\chi \in \chi} \sum_{y \in \gamma} p(x, y) \log _{2} \frac{p(x, y)}{p(x) \cdot p(y)}$

where $p(x), p(y)$ are the marginal probabilities of $X$ and $Y$, respectively, and $p(x, y)$ of the joint probability distribution of these two variables. $\mathrm{X}$ is a discrete random variable with alphabet, $\chi$, and probability density function $\mathrm{p}(\mathrm{x})=\operatorname{Pr}\{\mathrm{X}=\mathrm{x}\}$, $\mathrm{x} \in \mathrm{X}$ and $\mathrm{Y}$ is a discrete random variable with alphabet, $\gamma$, and probability density function $p(y)=\operatorname{Pr}\{Y=y\}, \mathrm{y} \in$. Thus, the mutual information $\mathrm{I}(\mathrm{X} ; \mathrm{Y})$ is the reduction in the uncertainty of $X$ due to the knowledge of $Y$ (Cover and Thomas 2006).

Nichols (2006) and Alonso et al. (2007) examined dynamic structures based on the delay $y_{n-\tau}$ with respect to $x_{n}$ of the mutual information. The DMI quantifies the dependence between random variables by obtaining the information shared on the basis of the displacement time, $\tau$, between $\mathrm{X}$ and $\mathrm{Y}^{\tau}$. Assuming that $X$ and $Y^{\tau}$ are stationary signals that depend on the joint probability of the delay time, $\tau$, Eq. 3 describes the DMI, according to Nichols (2006):

$I\left(X ; Y^{\tau}\right)=\sum_{x_{n} \in \chi} \sum_{y_{n-\tau} \in \gamma} p\left(x_{n}, y_{n-\tau}\right) \log _{2} \frac{p\left(x_{n}, y_{n-\tau}\right)}{p\left(x_{n}\right) \cdot p\left(y_{n-\tau}\right)}$

where $\mathrm{Y}^{\tau}=\left(\mathrm{y}_{1-\tau}, \mathrm{y}_{2-\tau}, \ldots, \mathrm{y}_{\mathrm{n}-\tau}\right)$ are random variables and $p\left(x_{n}\right)$ and $p\left(y_{n-\tau}\right)$ are the probability density functions (pdf) of $\mathrm{X}$ and marginal $Y^{\tau}$, respectively. The joint $p d f$ is given by $p\left(x_{n}, y_{n-\tau}\right)$, which is a function of the delay time, $\tau$.

The peak value of the DMI quantifies the channel capacity (Proakis and Salehi 2008) which was tested by generating surrogate data with the same power spectra and amplitude distribution as the real and simulated data, but with values 
randomized in time to make the signals independent (i.e., with zero mutual information). The surrogate algorithm implemented was based on that of Venema et al. (2006) as a Stochastic Iterative Amplitude Adjustment process shown in Fig. 2b.

The estimates of DMI from the surrogate data represent the values expected when the input and output do not share any connectivity (Fig, 2a, example in red). We fitted a Gaussian distribution to the 30 surrogate data estimates at $\mathrm{t}=\tau$ and thus obtained critical values at the $97 \%$ probability level, which were compared with DMI estimates from the recorded neural data.

\subsection{Cross-correlation for association between two variables}

Cross-correlation was used to identify the sign of interaction between neurons that were underpinned by inhibitory and excitatory linear interactions. We used cross-correlation between the GWN input and the neural response (spikes and synaptic potentials) to analyze the sign of the interactions. This measures the strength of the linear relationship between two variables (Therrien 1992), and the sign of the correlation coefficient.

\subsection{Channel capacities}

Channel capacity is defined (from Cover and Thomas 2006) as:

Fig. 2 Data analysis and significance levels. a. Algorithms for estimating the mutual information between input and output signals were tested on real and simulated biological data and significance levels tested at $97 \%$ against random probabilities generated from surrogate data with the same power spectra and amplitude distribution as real data. Analysis of one nonspiking local interneuron revealed a peak in DMI at $t=\tau$ with an amplitude dependent on channel capacity. $\mathbf{b}$. The iterative process to generate surrogate data with the same power spectra and amplitude distribution as real biological data
$C=\max I(X ; Y)$

where $C$ is computed in bits. The estimation of transmission rate, $R$, [bits/s] is based on channel capacity, $C$, from ShannonHartley Law (Proakis and Salehi 2008):

$R=2 \cdot B W \cdot C$

where $B W$ is the bandwidth in $H z$ [1/sec] and was evaluated based on rise time, according to Levine (1996):

$B W . T_{\text {rise }}=0.35$

From the data set, we estimate that $T_{\text {rise }}$ is approximately $50 \mu \mathrm{s}$. The test for spurious association was based on the significance level for surrogate data and a power test (Schreiber and Schmitz 2000). The relationship between the number of surrogate data $(\mathrm{N})$ and the probability of false rejection $(\alpha)$, corresponding to the level of significance (Therrien 1992) is:

$N=\frac{K}{\alpha}-1$

where $K=1$ for a one sided test. For $\alpha=0.03$ and $N=30$ we used $97 \%$ for the significance level of channel capacity.
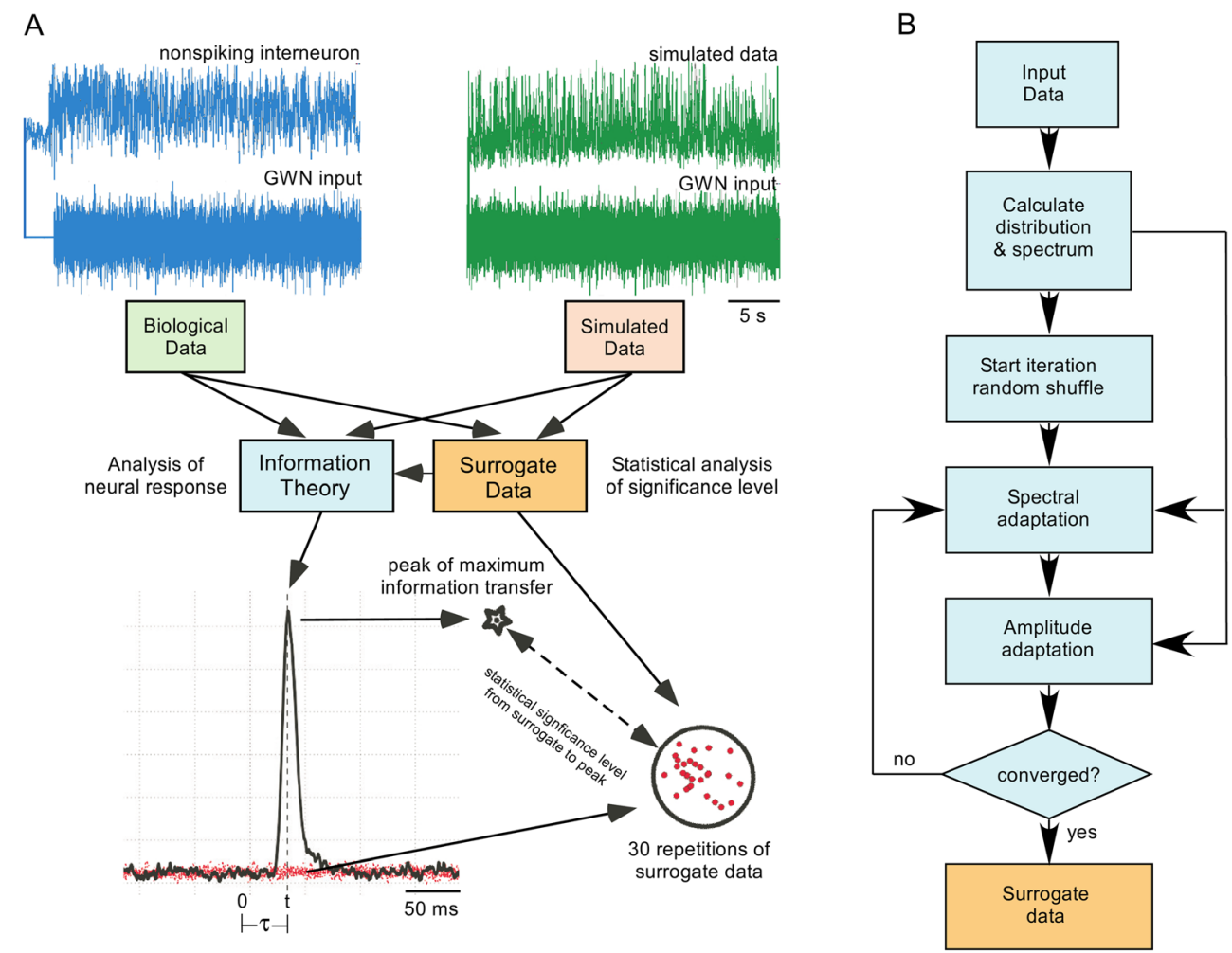


\subsection{Delayed mutual information}

From the recordings of neural responses we estimated the DMI (Fig. 2a), and from its peak value the delay and channel capacity. While the results presented here are based on biological data, the algorithms were also tested on simulated data (see Jin et al. 2010). Simulated data were generated from stochastic time series, $X_{t}$ and $Y_{t}$, based on:

$\mathrm{X}_{\mathrm{t}}=\mathrm{a}_{\mathrm{x}} \cdot \mathrm{X}_{\mathrm{t}-1}+\mathrm{n}_{\mathrm{X}}$

$Y_{t}=a_{y} \cdot Y_{t-1}+\zeta_{X Y} \cdot f\left(X_{t-1}\right)+n_{Y}$

where $n_{X}$ and $n_{Y}$ are independent Gaussian white noise. The level of coupling between the two synthetic time series is defined by $\zeta_{\mathrm{XY}}$ and the functional dependence of variables given by $\mathrm{f}(\mathrm{x})=x^{2}$. The level of coupling ranged in the interval $0.0 \leq \zeta_{\mathrm{XY}} \leq 1.0$, and the size of each time series ranged from eight to one hundred thousand samples. We then analysed the data to determine the levels of mutual information which gave rise to delayed peaks of mutual information at $\mathrm{t}=\tau$, with amplitudes depending on channel capacity (Fig. 2a). To test the significance of these peaks we generated surrogate data with the same power spectra and amplitude distribution as the real data (Fig. 2b). The surrogate algorithm implemented was based on that of Venema et al. (2006) as a Stochastic Iterative Amplitude Adjustment process. The significance of peaks in the estimates of DMI were tested against surrogate data.

Both biological and surrogate data were used to test the significance of the delayed mutual information peaks. As the surrogate data were not correlated with the system input, they represented bias and noise association (shown in red in Fig. 2a). We fitted a Gaussian distribution to the surrogate data at $\mathrm{t}=\tau$ and statistically compared that to the likelihood that a peak in DMI would occur with a $97 \%$ probability. We selected this high significance level to ensure accurate predictions of connectivity.

\section{Results}

\subsection{Local networks controlling limb movement}

Movements of the tibia relative to the femur of a hind leg of the locust are detected by a proprioceptor, the femoral chordotonal organ $(\mathrm{FeCO})$ located within the distal end of the femur and whose apodeme is stretched when the tibia is flexed (Fig. 1a) (Usherwood et al. 1967). The FeCO is responsible for mediating reflex movements of the leg. The approximately 90 sensory neurons encoding the dynamics of the movement of the tibia (Kondoh et al. 1995; Matheson 1990) embedded in the $\mathrm{FeCO}$ project to the metathoracic ganglion that contains the local networks that produce and control the movements of the leg. These neural networks (Fig. 1b) contain a number of layers of local interneurons that communicate by both digital signalling, the spiking local interneurons (Fig. 1c), and by analogue signalling, the nonspiking local interneurons (Fig. 1d) that generate graded potentials that in turn control sets of motor neurons (Burrows 1996). Other spiking intersegmental interneurons have axons and project to adjacent ganglia in the segmental chain (Newland 1990) coordinating the local networks for each leg.

\subsection{Nonspiking local interneurons}

The algorithm was applied to the synaptic responses of the nonspiking interneurons. Analyses of the dataset revealed interneurons with three distinct time delays in mutual information between mechanical excitation of the $\mathrm{FeCO}$ and the neuronal responses. In the first group (11 out of 36 interneurons recorded) (Fig. 3ai), interneurons had mean delays of mutual information of $14.2 \pm 0.4 \mathrm{~ms}$ (mean \pm SEM) (Fig. 3b). These delays were well defined and peaks in DMI were significant (at the 97\% level) when compared with surrogate data. A second group (Fig. 3aii) included 17 interneurons that exhibited peaks of mutual information at $25.7 \pm 1.3 \mathrm{~ms}$. A third group containing 8 interneurons, (Fig. 3aiii) had two pronounced peaks of DMI occurring at $31.8 \pm 0.9 \mathrm{~ms}$ and $45.3 \pm$ $1.5 \mathrm{~ms}$ (Fig. 3c). The peaks in DMI of the second and third groups were again found to be significant at the $97 \%$ level when compared to surrogate data (Schreiber and Schmitz 1996).

To determine the rate of information transfer of the interneurons we analyzed their channel capacities (Proakis and Salehi 2008) and found that they fell into three distinct categories corresponding to each time delay. Interneurons in the first group had mutual information of $7.2 \pm 1.5\left(\times 10^{-2}\right)$ bits, while in the second and third groups the mutual information were found to be $6.0 \pm 2.2\left(\times 10^{-2}\right)$ and $5.5 \pm 0.7\left(\times 10^{-2}\right)$ bits respectively (statistically significant at the $97 \%$ level). Assuming a bandwidth transmission of $350 \mathrm{~Hz}$ based on slew rate and rise time of the neural signals (Levine 1996) and channel capacities in bits/s per bandwidth (Proakis and Salehi 2008), then the channel capacity of the first group of interneurons was $28.8 \pm 2.5 \mathrm{bits} / \mathrm{s}$, and for the second and third groups were $21.0 \pm 3.8 \mathrm{bits} / \mathrm{s}$ and $19.3 \pm 1.2 \mathrm{bits} / \mathrm{s}$, respectively (Fig. $3 \mathrm{c}$ ).

Given the different time delays and mutual information we asked whether the nonspiking local interneurons could be grouped into distinct clusters using a method based on the Bayesian Information Criterion (BIC). Analysis revealed that the nonspiking interneuron clustered into three distinct groups (Fig. 3d).

Previous detailed physiological studies have analyzed the synaptic connections between $\mathrm{FeCO}$ sensory neurons and nonspiking interneurons (Fig. 4) and revealed that there are three known pathways through which the sensory neurons 
Fig. 3 Analysis of nonspiking local interneurons. a.

Representative examples of DMI revealed in three different groups of interneurons (i-iii). b. Mean times to peak of mutual information in 36 nonspiking local interneurons. c. The channel capacities of each group of interneurons were estimated based on a bandwidth transmission of $350 \mathrm{~Hz}$, and declined with increasing time delay. d. A BIC analysis of all 36 interneurons revealed 3 distinct clusters of interneuron based on time delays and channel capacities

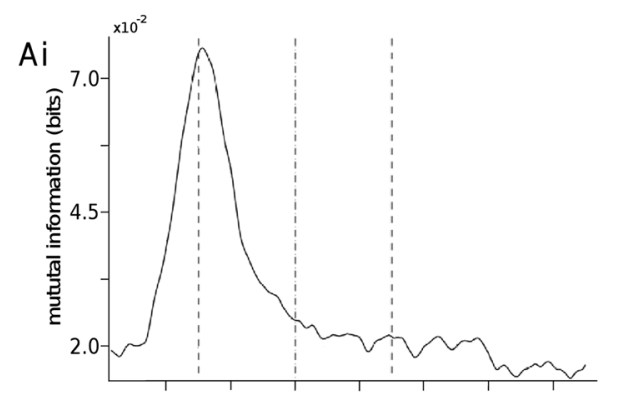

B
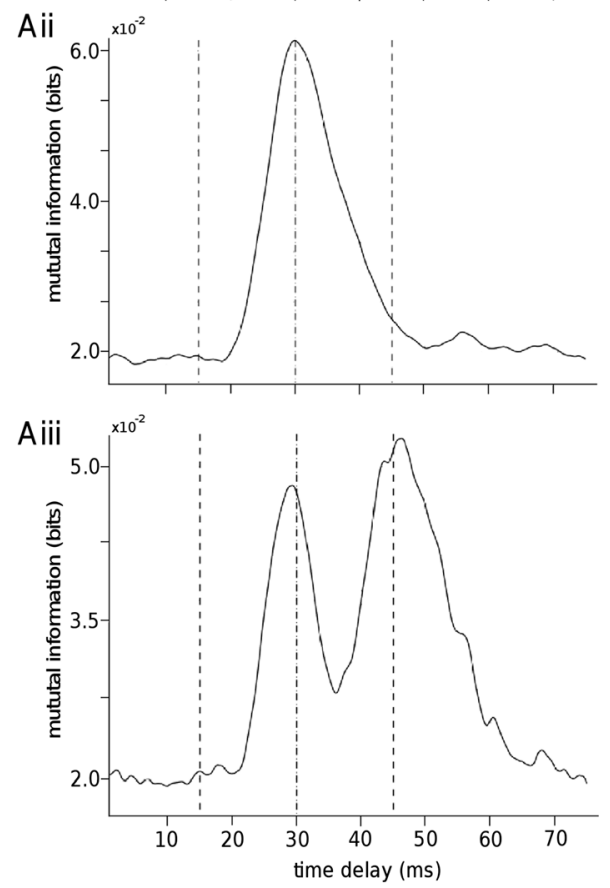

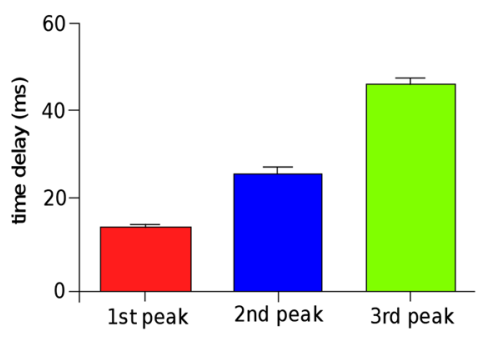

C
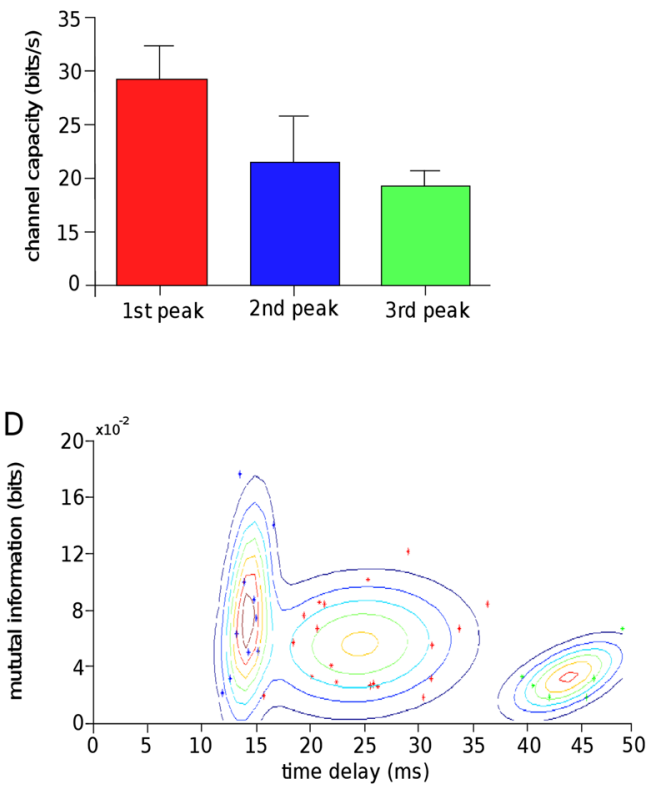

activate this class of interneuron. First, $\mathrm{FeCO}$ sensory neurons excite nonspiking local interneurons via monosynaptic cholinergic connections (Burrows et al. 1988). Second,

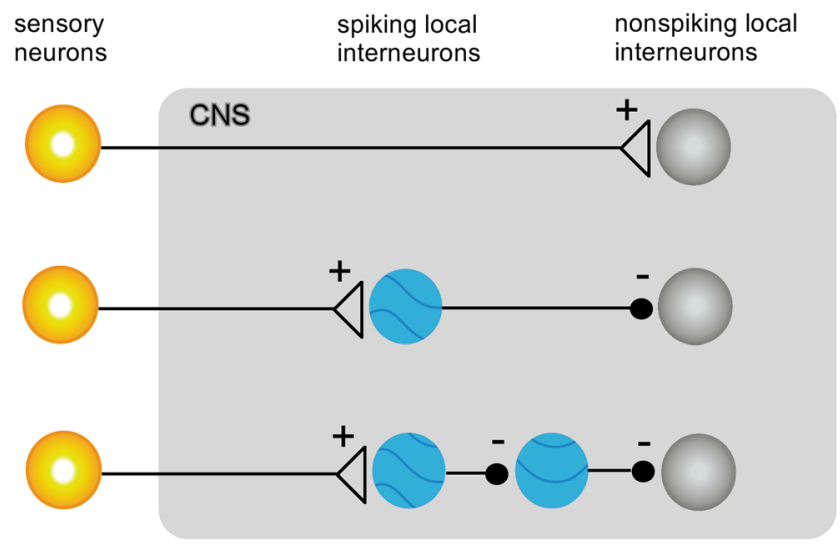

Fig. 4 Identified synaptic pathways between FeCO sensory neurons and nonspiking local interneurons (modified from Burrows et al. (1988)). Nonspiking interneurons can be excited directly by FeCO sensory neurons, inhibited indirectly through a single layer of GABAergic spiking local interneuron or dis-inhibited indi- rectly through two layers of GABergic spiking local interneurons nonspiking interneurons are inhibited by the $\mathrm{FeCO}$ sensory neurons via interposed spiking local interneurons from a midline population with GABAergic (Watson and Burrows 1987) inhibitory outputs onto the nonspiking interneurons (Burrows et al. 1988). A third known pathway results in an excitation of the nonspiking local interneurons through two layers of GABAergic spiking local interneurons, each with inhibitory outputs, thereby resulting in a disinhibition of the nonspiking interneurons (Burrows et al. 1988).

\subsection{Spiking local interneurons}

We then tested the hypothesis that the different groups of nonspiking local interneurons found based on DMI were related to the known synaptic pathways by analyzing the synaptic responses of a midline population of spiking local interneurons. The midline spiking local interneurons have been studied in extraordinary detail, from their integration of sensory signals and their output effects during a range of movements (Burrows et al. 1988), to the arrangement of their input and output synapses (Watson and Burrows 1987) and their dynamics (Vidal-Gadea et al. 2010). 
We analysed the synaptic responses of 25 spiking local interneurons, which revealed that they also showed clearly defined peaks of DMI (Fig. 5a), that occurred with two distinct mean delays in different interneurons of $17.0 \pm 1.3 \mathrm{~ms}$ and $29.2 \pm 1.1 \mathrm{~ms}$ (Fig. 5b) and with mutual information of $2.7 \pm$ $0.6\left(\times 10^{-2}\right)$ bits and $1.8 \pm 0.2\left(\times 10^{-2}\right)$ bits. Again, by assuming a bandwidth of $350 \mathrm{~Hz}$, the channel capacity of the first group of spiking local interneurons was $9.5 \pm 1.9 \mathrm{bits} / \mathrm{s}$ and in the second group $6.3 \pm 0.6$ bits/s (Fig. 5c). A subsequent BIC analysis confirmed that the spiking local interneurons grouped into two distinct clusters (Fig. 5d) based on the time delay and mutual information.

Furthermore, we compared the time delays to the first 2 peaks of the nonspiking interneurons with those of the spiking local interneurons. Given that both classes of interneuron receive monosynaptic cholinergic inputs (Burrows 1996) from the FeCO sensory neurons it would be expected that the times to the positive, excitatory, first peak of each interneuron would not be different. The mean time to first peak in the nonspiking interneurons was $14.22 \pm 0.46 \mathrm{~ms}$, whereas that to the first peak of the spiking interneurons was $17.04 \pm 1.3 \mathrm{~ms}$. A students $T$ test indicated that these two peak times were not significantly different $(\mathrm{t}=1.765$, d.f. $=22, P<0.05)$, as would be expected if they were both generated by monosynaptic inputs.

The second negative, inhibitory, peak of each class of interneuron is hypothesized to be mediated by a single layer of GABAergic inhibitory spiking interneurons. Thus the times to second peak would not be expected to differ between the two types of interneuron. Results show that the mean time to second peak in the nonspiking interneurons was $26.23 \pm 1.31 \mathrm{~ms}$, $n=16$, whereas that to the second peak of the spiking interneurons was $29.36 \pm 1.09 \mathrm{~ms}, n=16$. A students $T$ test indicated that these two peak times were not significantly different $(\mathrm{t}=1.836$, d.f. $=30, P<0.05)$, as would be expected if they
Fig. 5 Analysis of spiking local interneurons. a. Examples of delayed mutual information revealed in two different interneurons (i-ii). b. Mean times to peak of mutual information in 25 spiking local interneurons. c. Interneurons with the shortest delayed mutual information had the highest channel capacities (based on a bandwidth of $350 \mathrm{~Hz}$ ). d. A BIC analysis revealed two distinct clusters of interneuron based on time delay and channel capacity

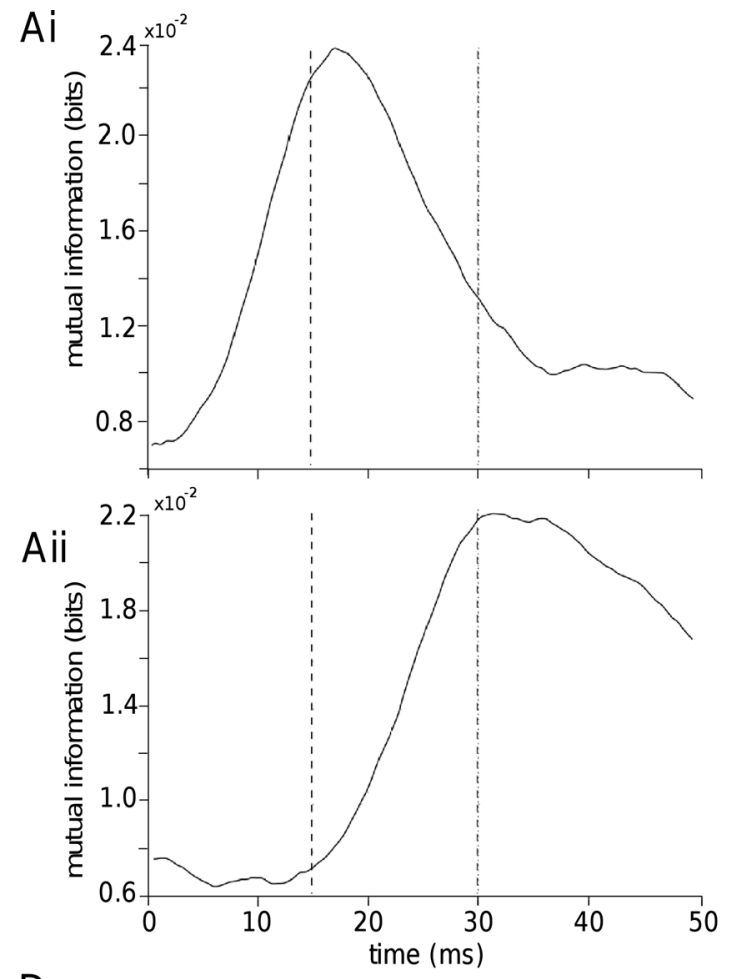

D

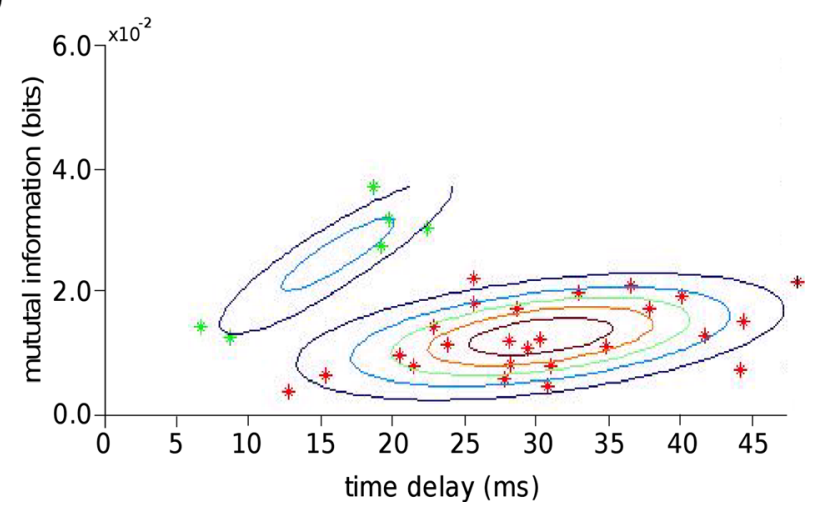

B
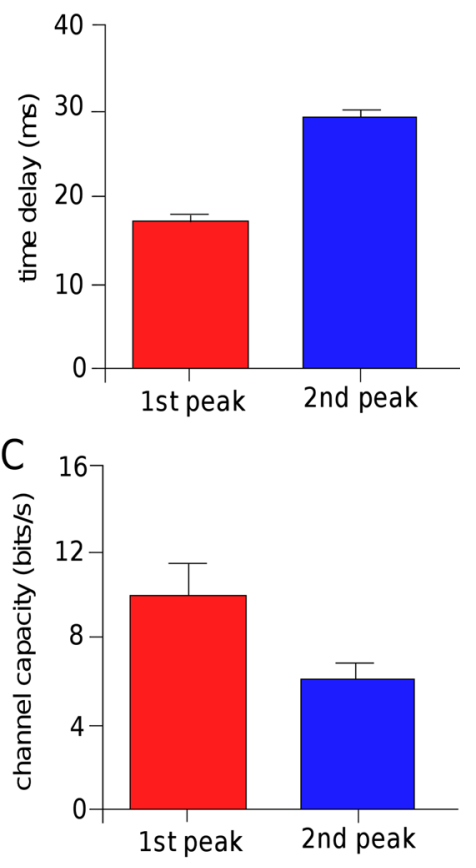
were both mediated through a single layer of interposed inhibitory spiking local interneurons.

\subsection{Sensory neurons}

Further confirmation that the peaks in mutual information were related to position in the neural network were obtained by analyzing the response properties of the $\mathrm{FeCO}$ sensory neurons. Given technical difficulties related to recording their intracellular responses from their somata in the $\mathrm{FeCO}$ their spikes are commonly recorded in their axons as they enter the metathoracic ganglion (Matheson 1990). We recorded the spike responses of a small subset $(n=12)$ of the sensory neurons while moving the $\mathrm{FeCO}$ apodeme with a GWN waveform (Fig. 6a).

Analysis of the mutual information of the sensory neurons revealed a single peak occurring with a mean time delay of $12.8 \pm 0.5 \mathrm{~ms}$, with a mean of mutual information of $11.2 \pm 1.8$

\section{A}

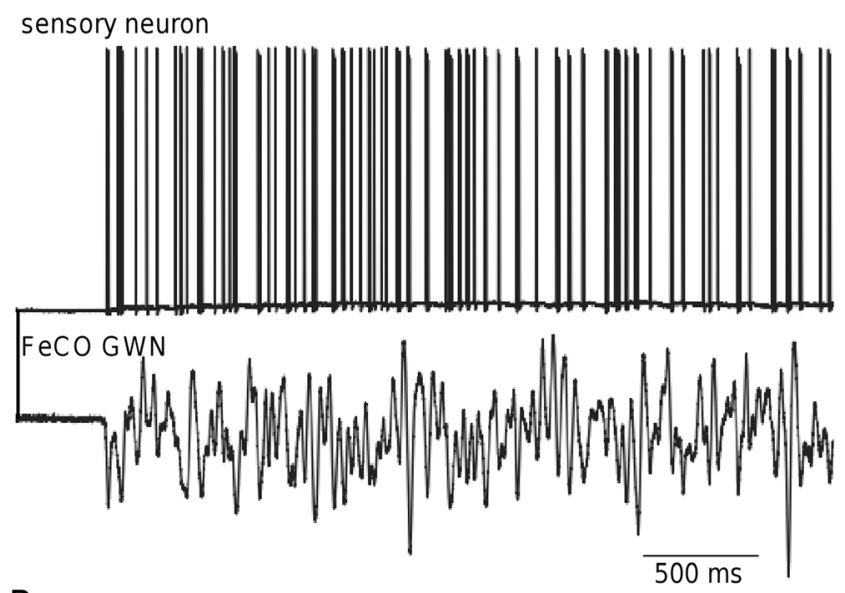

B

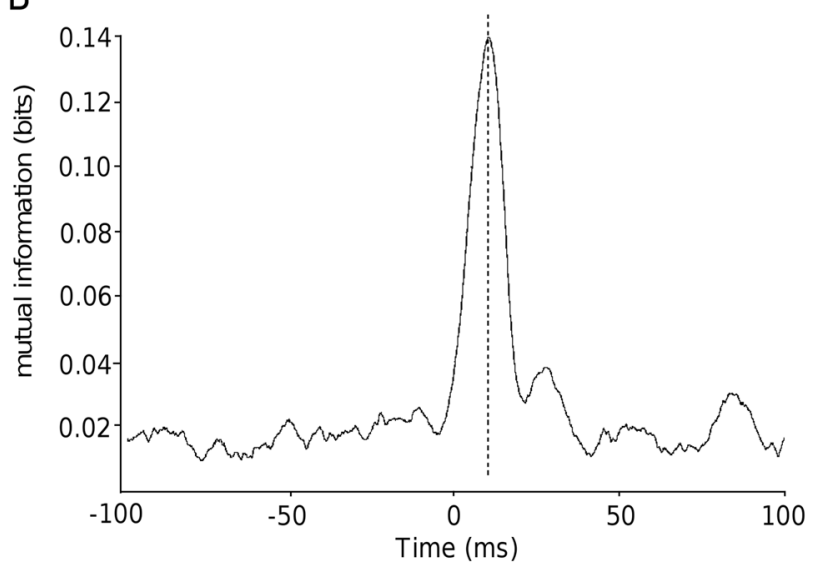

Fig. 6 Delayed mutual information in sensory neurons of the FeCO. a. Intracellular recording of the spike activity of a sensory neuron recorded in its axon in nerve 5, just as it enters the metathoracic ganglion. The apodeme of the FeCO was moved with a GWN signal with a cut off frequency of $27 \mathrm{~Hz}$. b. An example of a peak of delayed mutual information in a sensory neuron with a time to peak of $11 \mathrm{~ms}$ $\left(\times 10^{-2}\right)$ bits (mean \pm SEM, $\left.n=12\right)$ and channel capacity of $39.2 \pm 3.1 \mathrm{bits} / \mathrm{s}$.

\subsection{Sign of connections}

Further evidence to support the hypothesis that the delays in mutual information were related to the number of layers in the network preceding the neuron being studied was sought by identifying the polarity of the interactions, i.e., whether the interactions were excitatory or inhibitory. As indicated above, DMI does not identify the sign of the association between signals and hence we carried out a cross-correlation between the GWN and the responses of the sensory neurons and local interneurons to establish the sign of connections (Fig. 7). We found that, for sensory neurons, the peak in correlation.

was positive in all 12 sensory neurons tested, while for the spiking local interneurons, the first peak was positive in $100 \%$ (9 from 9 interneurons) of all neurons with significant peaks in mutual information $(n=12)$, while the second peak was negative in $60 \%$ of interneurons ( 9 from 16 interneurons). For the nonspiking interneurons the first peak was positive in $64 \%$ of interneurons ( 7 from 11 interneurons), negative for the second peak in $76 \%$ of interneurons (13 from 17 interneurons) and positive for the third peak in $75 \%$ of tests $(6$ from 8 interneurons).

\section{Discussion}

A number of neural networks in both vertebrates (Alle and Geiger 2006) and invertebrates (Büschges 1990; Burrows 1996) function with neurons that do not spike. While an understanding of how these circuits are anatomically organized has been pursued for many years, particularly in the invertebrates, few studies have utilized the benefits of computational approaches to better understand their connectivity. We asked whether information theoretic approaches could provide insights into neuron connectivity within multi-layered networks with a complex input and output organization. In particular we focused on a well-studied and understood local network responsible for producing and controlling the movements of the tibia about the femur of the hind leg of the locust. By analysing the synaptic potentials of different classes of interneurons we found distinct peaks in DMI whose channel capacities declined with the time delay to peak. Nonspiking local interneurons fell into three distinct clusters following a cluster analysis using Bayesian Information Criterion (BIC) based on channel capacity and time delay, whereas spiking local interneurons fell into two distinct categories. 
Fig. 7 Cross-correlation reveals polarity of connections of neurons in a local network. a. The peak in mutual information on the sensory neuron was always positive (excitatory) in all 12 sensory neurons tested. $\mathbf{b}$. In spiking local interneurons the first peak was always positive (excitatory) but the second peak was negative (inhibitory) in most interneurons. c. In nonspiking interneurons the first peak was generally positive, the second negative and the third positive (excitatory). Arrowheads indicate the times of peak DMI.

Correlation coefficients of 1 and $n 1$ represent maximum correlation
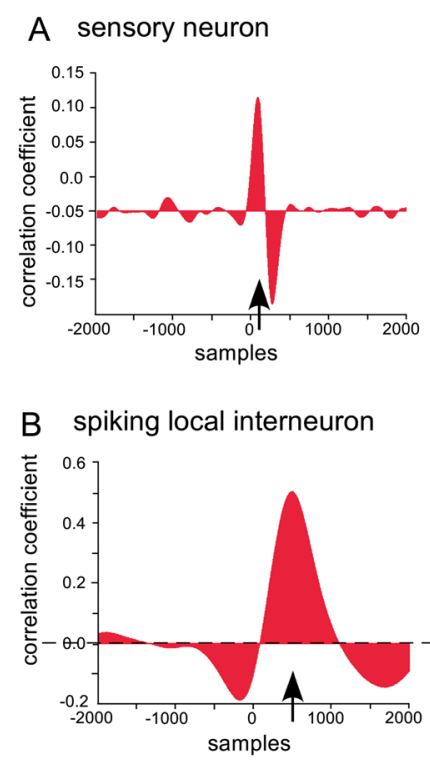

C nonspiking local interneuron
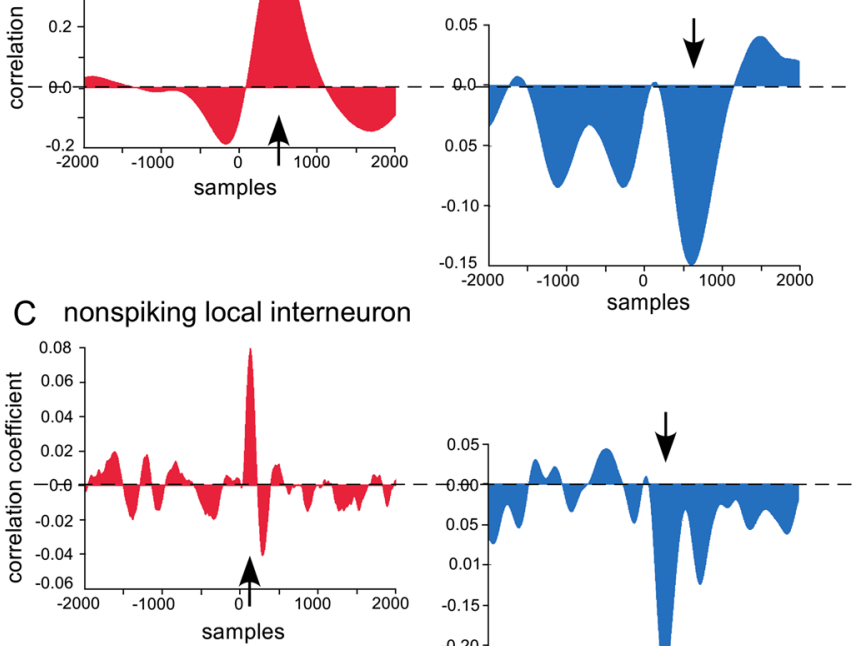

1st peak
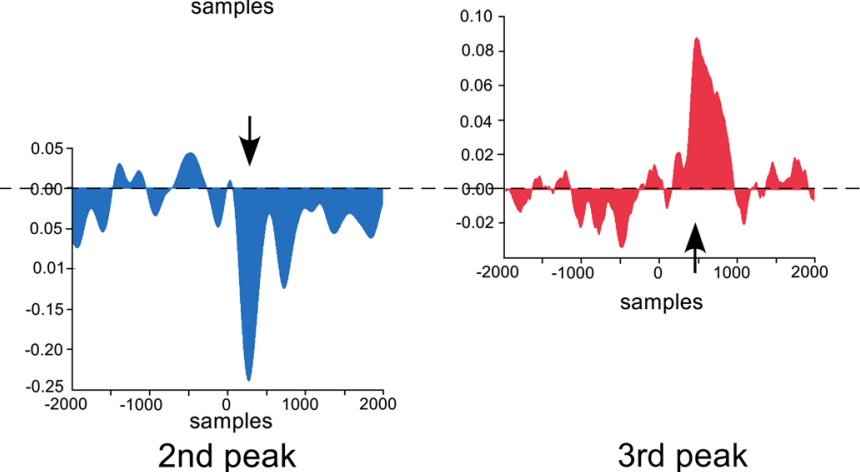

3rd peak

\subsection{Interpretation of time delays and channel capacities}

Burrows (1987), in a detailed analysis of the properties of synaptic inputs to spiking local interneurons, suggested that excitatory $\mathrm{FeCO}$ inputs onto a midline population of were monosynaptic and chemically mediated. He also showed that the minimum time to evoke a reflex response was approximately $13 \mathrm{~ms}$ for afferents with the fastest conduction velocities of $4 \mathrm{~m} \cdot \mathrm{s}^{-1}$, allowing for conduction of the afferent spike from the $\mathrm{FeCO}$ to the nervous system, a central synaptic delay and activation of a motor neuron. Approximately $10 \mathrm{~ms}$ of this time is taken simply to the start of an EPSP in a motor neuron. A similar delay of approximately $10 \mathrm{~ms}$ occurs to monosynaptic EPSPs in spiking local interneurons (Burrows 1987) and nonspiking local interneurons (Burrows et al. 1988). Given that proprioceptive afferents have conduction velocities from $2.8-4 \mathrm{~m} \cdot \mathrm{s}^{-1}$ considerably longer delays might be expected (Burrows 1987) for sensory afferents with lower conduction velocities. Since the FeCO afferents make monosynaptic connections with both classes of interneuron it would be reasonable to conclude from the synaptic delays given above that the information transfer times would be of the same order of magnitude, which indeed is what we have found in this study with peaks of mutual information with delays of $17.0 \pm 1.3 \mathrm{~ms}$ for spiking interneurons and $14.2 \pm 0.4 \mathrm{~ms}$ for nonspiking interneurons.

Given the patterns of connection illustrated here in Fig. 4, the work of Burrows (1987) also provides us with clues as to the origin of the second peaks in both classes of interneuron and the third peak in nonspiking interneurons. He showed that the time from initial depolarization to a spike in a spiking local interneuron is approximately $10-14 \mathrm{~ms}$. Allowing for a further $0.5-1.0 \mathrm{~ms}$ for conduction of the spike within the cell to its output synapses and a further $0.5-1.5 \mathrm{~ms}$ for central synaptic delay then we would expect a further 11-16.5 ms for each layer of spiking local interneurons in the network. Indeed these synaptic delays match well with the observed mutual information delays found here.

On this basis our working hypothesis is that the delays in mutual information in an interneuron are related to its position in a network. Support for this hypothesis comes from information regarding the sign of interactions between the different 
layers of interneurons. Proprioceptive afferents make excitatory monosynaptic connections with both spiking (Burrows 1987) and nonspiking interneurons (Burrows et al. 1988). Thus the first peaks in mutual information of both types of interneuron would be expected to be excitatory. Our crosscorrelation analysis showed that $100 \%$ of the first peaks of mutual information of spiking interneurons are excitatory while $64 \%$ of the first peaks in nonspiking interneurons are excitatory. The second peak of the spiking and nonspiking interneurons could be accounted for by inhibitory GABAergic interneurons that are known to be interposed between these closes of interneuron (Burrows 1996) that would mean that these second peaks would be inhibitory in nature. Our crosscorrelation analysis also predicted that $76 \%$ of second peaks of nonspiking interneurons and $60 \%$ of spiking interneurons would be inhibitory. Finally, the third peak in mutual information in nonspiking interneurons could be produced by two layers of GABAergic inhibitory interneurons (Burrows et al. 1988) resulting in a disinhibition of the nonspiking interneurons. Our cross correlation analysis again predicts the third peak results from a positive interaction in $75 \%$ of interneurons.

We should however consider why these probabilities are not all at the $100 \%$ level. Part of the problem resides in the fact we do not yet know all of the pathways between layers of interneurons, or that others also have a major contribution. While Burrows et al. (1988) described the potential pathways that existed between sensory neurons and nonspiking interneurons, an earlier study by Burrows (1979) showed that there are synaptic interactions between nonspiking local interneurons themselves so that one nonspiking interneuron can exert a graded control over the membrane potential of another. Synaptic connections between interneurons are principally inhibitory and one way (Burrows 1996). Given that the nonspiking interneurons can have both inhibitory GABAergic outputs (Watson and Burrows 1987; Wildman et al. 2002) and excitatory outputs then it is likely that the second peak of the nonspiking interneurons could also be positive in some nonspiking interneurons. Indeed our results indicate that in $18 \%$ of nonspiking interneurons there are positive interactions at the level of the second peak. This in turn would have effects on downstream interneurons. Such complexity in the pathways may therefore underlie the known dynamic groups of nonspiking interneurons described by Angarita-Jaimes et al. (2012). Thus our results not only predict the known patterns of connectivity already established so far but point to further interneuron interactions that have yet to be demonstrated at the physiological level.

\subsection{Nonspiking communication}

Nonspiking communication has been known for many years in invertebrates and is found at many levels in neural networks, from sensory neurons in crabs (DiCaprio 2004) to interneurons in crustaceans (Nagayama et al. 1984; Samarandache-Wellmann et al. 2013) and insects (Burrows 1996; Büschges 1990). In recent years it has become apparent that nonspiking communication is an important component of effective network action in vertebrates as well. For example, Alle and Geiger (2006) described spiking (digital) and analogue communication in hippocampal mossy fibres, while Shu et al. (2006) showed that synaptic signaling results from a mixture of spikes and analogue signals that modulate the amplitude and duration of action potentials through $\mathrm{Ca}^{2+}$ dependent mechanisms.

It is thought that the advantage of analogue transmission resides in the increased bit rate afforded by such transmission (Laughlin et al., 1998) although over long distances this is not the case. For example, Dicaprio (2004) showed that transmission in nonspiking stretch receptors in the crab was over 2,500 bits/s, however this declined sharply with conduction distance. Here we find that transfer rates are several orders of magnitude lower, potentially due to the energy demands of signal transfer (Laughlin et al. 1998) but also due to their function in orchestrating sets of motor neurons to produce appropriate movements of the limb, where digital signalling would require many more neurons to generate a smooth and continuous control over motor neuron activity.

A study by de Ruyter van Steveninck and Laughin (1996) presents the rates of information transfer for spiking and nonspiking neurons in the visual system of the blowfly. Comparing transmission rates in pre- and post-synaptic cells, they estimate that each synaptic active zone transmits at approximately 50bits/s and that the graded responses of nonspiking cells transmit at a rate five times higher than spiking interneurons. Our results also show a higher rate of information transfer in nonspiking compared to spiking interneurons in a proprioceptive network, in a range similar to that described by DiCaprio (2004).

\subsection{Computational models and network inference}

It is now clear that there is no single universal approach to understanding connectivity in the brain (Amblard and Michel 2011) and approaches extend from non-directional measures such as mutual information (Jirsa and McIntosh 2007) to measures that utilize directional information flow such as transfer entropy (Lungarella and Sporns 2006). Lungarella and Sporns (2006) show that there is a distinct link between the morphology of sensors and information flow and that coding depends on the morphology and dynamics of sensory systems. Topographical maps are commonplace in the neural networks of many animals, including the locust (Burrows and Newland 1993; Matheson 1990; Newland 1991), and methods that incorporate these features of networks, and their network dynamics (Vidal-Gadea et al. 2010), are crucial to understand 
better neural network function. Gerhard et al. (2013) developed a network inference algorithm in a relatively simple three motor neuron oscillatory network in the stomatogastric ganglion of the crab. We go a step further and use computational approaches to reveal pathways of information flow between layers of interneurons in a more complex neural network that utilises both digital and analogue signaling and show that this method achieves a high degree of inference about connectivity.

The current paper demonstrates the promise of this computational approach leading to results highly compatible with those obtained from previous physiological studies, and thus serves to independently confirm their results. The method also demonstrates the potential of this approach to gain additional insights into more complex connectivity patterns, based on relatively simple stimulation and recording procedures.

Acknowledgements The authors are grateful to Vincent O'Connor and John Chad for their comments on an earlier draft of the manuscript. PLN and DMN were supported through awards from The Biotechnology and Biological Sciences Research Council and the Engineering and Physical Sciences Research Council (United Kingdom) and CDM was supported through an award from the Research Foundation of Brazil (CNPq). PLN and CDM were supported by an award from the Coordenação de Aperfeiçoamento de Pessoal de Nível Superior (CAPES).

Conflict of interest The authors declare that they have no conflict of interest.

\section{References}

Alle, H., \& Geiger, J. R. P. (2006). Combined analog and action potential coding in Hippocampal mossy fibers. Science, 311(5765), 1290 1293. doi:10.1126/ science. 1119055.

Alonso, J. F., Mananas, M. A., Topor, D. H. Z. L., \& Bruce, E. N. (2007). Evaluation of respiratory muscles activity by means of cross mutual information function at different levels of ventilatory effort. IEEE Transactions on Biomedical Engineering, 54(9), 1573-1582.

Amblard, P. O., \& Michel, O. J. J. (2011). On directed information theory and granger causality graphs. Journal of Computational Neuroscience, 30(1), 7-16.

Angarita-Jaimes, N., Dewhirst, O. P., Simpson, D. M., Kondoh, Y., Allen, R., \& Newland, P. L. (2012). The dynamics of analogue signaling in local networks controlling limb movement. The European Journal of Neuroscience, 36(9), 3269-3282. doi:10.1111/j.1460-9568.2012. 08236.x.

Bialek, W., Nemenman, I., \& Tishby, N. (2001). Predictability, complexity and learnings. Neural Computation, 13(11), 2409-2463. doi:10. 1162/089976601753195969.

Bressler, S. L., \& Seth, A. K. (2011). Wiener-granger causality: a well established methodology. NeuroImage, 58(2), 323-329. doi:10. 1016/j.neuroimage.2010.02.059.

Burrows, M. (1979). Graded synaptic interactions between local premotor interneurons of the locust. Journal of Neurophysiology, 42(4), 1108-1123.

Burrows, M. (1987). Parallel processing of proprioceptive signals by spiking local interneurons and motor neurons in the locust. The Journal of Neuroscience, 7, 1064-1080.
Burrows M (1996) The Neurobiology of an Insect Brain. Oxford University Press, UK, ISBN-13: 978-0198523444

Burrows, M., \& Newland, P. L. (1993). Correlation between the receptive fields of locust interneurons, their dendritic morphology, and the central projections of mechanosensory neurons. The Journal of Comparative Neurology, 329(3), 412-26.

Burrows, M., Laurent, G., \& Field, L. H. (1988). Proprioceptive inputs to nonspiking local interneurons contribute to local reflexes of a locust hindleg. The Journal of Neuroscience, 8, 3085-3093.

Büschges, A. (1990). Nonspiking pathways in a joint-control loop of the stick insect carausius morosus. The Journal of Experimental Biology, 151, 133-160.

Cover, T. M., \& Thomas, J. A. (2006). Elements of information theory (2nd ed.). Hoboken: Wiley.

Dewhirst, O. P., Angarita-Jaimes, N., Simpson, D. M., Allen, R., \& Newland, P. L. (2013). A system identification analysis of neural adaptation dynamics and nonlinear responses in the local reflex control of locust hind limbs. Journal of Computational Neuroscience, 34(1), 39-58. doi:10.1007/s10827-012-0405-9.

DiCaprio, R. A. (2004). Information transfer rate of nonspiking afferent neurons in the crab. Journal of Neurophysiology, 92(1), 302-310. doi:10.1152/jn.01176.2003.

Dionisio, A., Menezes, R., \& Mendes, D. A. (2004). Mutual information: a measure of dependency for nonlinear time series. Physica A, 344, 326-329.

Erdogmus, D., \& Principe, J. C. (2006). From linear adaptive filtering to nonlinear information processing. IEEE Signal Processing Magazine, 24(6), 14-33. doi:10.1109/SP-M.2006.248709.

Gerhard F, Kispersky T, Gutierrez GJ, Marder E, Kramer M, Eden U (2013) Successful reconstruction of a physiological circuit with known connectivity from spiking activity alone. PLoS Comp Biol 9(7), DOI10.1371/journal.pcbi.1002653

Hlavácková-Schindler, K., Palus, M., Vejmelka, M., \& Bhattacharya, J. (2007). Causality detection based on information-theoretic approaches in time series analysis. Physics Reports, 441(1), 1-46. doi:10.1016/j.physrep.2006.12.004.

Jin, S. H., Lin, P., \& Hallett, M. (2010). Linear and nonlinear information flow based on time-delayed mutual information method and its application to corticomuscular interaction. Clinical Neurophysiol, 121(3), 392-401. doi:10.1016/j.clinph. 2009.09.033.

Jirsa, V. K., \& McIntosh, A. R. (2007). Handbook of brain connectivityunderstanding complex systems. New York: Springer.

Kondoh, Y., Okuma, J., \& Newland, P. L. (1995). Dynamics of neurons controlling movements of a locust hind leg: wiener kernel analysis of the responses of proprioceptive afferents. Journal of Neurophysiology, 73(5), 1829-1842.

Laughlin, S. B., De Ruyter Van Steveninck, R. R., \& Anderson, J. C. (1998). The metabolic cost of neural information. Nature Neuroscience, 1(1), 36-41. doi:10.1038/236.

Levine, W. S. (1996). The control handbook. Boca Raton: CRC Press.

$\mathrm{Li}, \mathrm{W}$. (1990). Mutual information functions versus correlation functions. Journal of Statistical Physics, 60(5-6), 823-837.

Li, X., \& Ouyang, G. (2010). Estimating coupling direction between neuronal pop- ulations with permutation conditional mutual information. NeuroImage, 52(2), 497-507. doi:10.1016/j.neuroimage. 2010.05.003.

Lungarella M, Sporns O (2006) Mapping information flow in sensorimotor networks. PLoS Comp Biol 2(10), DOI http://dx.doi.org/10. 1371/journal. pcbi.0020144 DOI:10.1371/journal.pcbi.0020144

Mars, N., \& van Arragon, G. (1982). Time delay estimation in non-linear systems using average amount of mutual information analysis. Signal Processing, 4(2-3), 139-153. doi:10.1016/0165-1684(82) 90017-2.

Marschinski, R., \& Kantz, H. (2002). Analysing the information flow between financial time series. Europ Phys J B - Condensed Matter 
and Complex Systems, 30, 275-281. doi:10.1140/epjb/e200200379-2.

Matheson, T. (1990). Responses and locations of neurones in the locust metathoracic femoral chordotonal organ. Journal of Comparative Physiology. A, 166(6), 915-927. doi:10.1007/BF00187338.

Müller, T., Lauk, M., Reinhard, M., Hetzel, A., Lücking, C., \& Timmer, J. (2003). Estimation of delay times in biological systems. Annals Biomedical Engineering, 31(11), 1423-1439. doi:10.1114/1. 1617984.

Nagayama, T., Takahata, M., \& Hisada, M. (1984). Functional characteristics of local non-spiking interneurons as the pre-motor elements in crayfish. Journal of Comparative Physiology. A, 154(4), 499-510. doi:10.1007/BF00610164.

Newland, P. L. (1990). Morphology of a population of mechanosensory ascending interneurones in the metathoracic ganglion of the locust. The Journal of Comparative Neurology, 299(2), 242-260. doi:10. 1002/cne.902990208.

Newland, P. L. (1991). Morphology and somatotopic organisation of the central projections of afferents from tactile hairs on the hind leg of the locust. The Journal of Comparative Neurology, 312(4), 493 508 .

Newland, P. L., \& Kondoh, Y. (1997a). Dynamics of neurons controlling movements of a locust hind leg II. flexor tibiae motor neurons. Journal of Neurophysiology, 77(4), 1731-1746.

Newland, P. L., \& Kondoh, Y. (1997b). Dynamics of neurons controlling movements of a locust hind leg III. extensor tibiae motor neurons. Journal of Neurophysiology, 77(6), 3297-3310.

Nichols, J. (2006). Examining structural dynamics using information flow. Probabilistic Engineering Mechanics, 21(4), 420-433.

Nichols, J. M., Seaver, M., Trickey, S. T., Todd, M. D., Olson, C., \& Overbey, L. (2005). Detecting nonlinearity in structural systems using the transfer entropy. Physical Review E, 72, 046,217. doi:10. 1103/PhysRevE.72.046217.

Palus, M., Komárek, V., \& Sterbová, Z. H. K. (2001). Synchronization as adjustment of information rates: detection from bivariate time series. Physical Review E, 63, 046211. doi:10.1103/PhysRevE.63.046211.

Pearl J (2009) Causal inference in statistics: An overview. Statistics Surveys 3:96-146 ISSN: 1935-7516, DOI:10.1214/09-SS057

Pérez, F., \& Granger, B. (2007). Ipython: a system for interactive scientific computing. Computing Science Engineering, 9(3), 21-29. doi: 10.1109/MCSE.2007.53.

Proakis, J. G., \& Salehi, M. (2008). Digital communications. New York: McGraw-Hill Higher Education.

Schreiber, T. (2000). Measuring information transfer. Physical Reviews Letters, 85(2), 461-464. doi:10.1103/PhysRevLett.85.461.

Schreiber, T., \& Schmitz, A. (1996). Improved surrogate data for nonlinearity tests. Physical Reviews Letters, 77, 635.

Schreiber, T., \& Schmitz, A. (2000). Surrogate time series. Physica D, 142(3-4), 346-382. doi:10.1016/S0167-2789(00)00043-9.
Shannon, C. E. (1948). A mathematical theory of communication. Bell System Technical Journal, 27(3), 379-423.

Shibuya T, Harada T, Kuniyoshi Y (2009) Causality quantification and its applications: Structuring and modeling of multivariate time series. In: Proceedings of the 15th ACM SIGKDD International Conference on Knowledge Discovery and Data Mining, ACM, New York, NY, USA, KDD’09, pp 787- 796, DOI 10.1145/ 1557019.1557106

Shu Y, Hasenstaub A, Duque A, Yu Y, McCormick DA (2006) Modulation of intracortical synaptic potentials by presynaptic somatic membrane potential. Nature pp 761-765, DOI http://dx.doi. org/10.1038/nature 04720

Sommerlade, L., Amtage, F., Lapp, O., Hellwig, B., Lücking, C. H., Timmer, J., \& Schelter, B. (2011). On the estimation of the direction of information flow in networks of dynamical systems. Journal of Neuroscience Methods, 196(1), 182-189. doi:10.1016/j.jneumeth. 2010.12.019.

Stetter O, Battaglia D, Soriano J, Geisel T (2012) Model-free reconstruction of excitatory neuronal connectivity from calcium imaging signals. PLos Comp Biol 8(8), DOI 10.1371/journal.pcbi.1002653

Therrien CW (1992) Discrete Random Signals and Statistical Signal Processing. Prentice Hall, NJ, United States of America

Usherwood, P. N. R., Runion, H. I., \& Campbell, J. I. (1967). Structure and physiology of a chordotonal organ in the locust leg. The Journal of Experimental Biology, 48, 305-323.

van Steveninck RR, D. R., \& Laughlin, S. B. (1996). The rate of information transfer at graded-potential synapses. Nature, 379, 642-645. doi: $10.1038 / 379642 \mathrm{a} 0$.

Vastano, J. A., \& Swinney, H. L. (1988). Information transport in spatiotemporal systems. Physical Reviews Letters, 60, 1773-1776. doi:10. 1103/PhysRevLett.60.1773.

Venema, V., Ament, F., \& Simmer, C. (2006). A stochastic iterative amplitude adjusted fourier transform algorithm with improved accuracy. Nonlinear Processes in Geophysics, 13(3), 321-328. doi:10. 5194/npg-13-321-2006.

Vidal-Gadea, A., Jing, X. J., Simpson, D. M., Dewhirst, O., Kondoh, Y., \& Newland, R. A. P. (2010). Coding characteristics of spiking local interneurons during imposed limb movements in the locust. Journal of Neurophysiology, 103, 603-615.

Ward, B. D., \& Mazaheri, Y. (2008). Information transfer rate in fMRI experiments measured using mutual information theory. $J$ Neurosci Meth, 167(1), 22-30. doi:10.1016/j.jneumeth.2007.06.027.

Watson, A. H. D., \& Burrows, M. (1987). Immunocytochemical and pharmacological evidence for gabaergic spiking local interneurons in the locust. The Journal of Neuroscience, 7, 174-1751.

Wildman, M., Ott, S. R., \& Burrows, M. (2002). Nonspiking pathways in a joint-control loop of the stick insect carausius morosus. The Journal of Experimental Biology, 205, 3651-3659. 